\title{
Modification of Breast Cancer Milieu with Chemotherapy Plus Dendritic Cell Vaccines: An Approach to Select Best Therapeutic Strategies
}

Luis Daniel Mejias Sosa ( $\square$ luis.mejiass@hospitalreyjuancarlos.es )

Hospital Universitario Rey Juan Carlos https://orcid.org/0000-0003-0927-6997

Álvaro López-Janeiro

Hospital Universitario La Paz

Alicia Córdoba

Complejo Hospitalario de Navarra

Pablo Sala

Clínica Universidad de Navarra

\section{Belén P Solans}

Universidad de Navarra Facultad de Farmacia: Universidad de Navarra Facultad de Farmacia y Nutricion

\section{Susana Inogés}

Clínica Universidad de Navarra

\section{Ascensión López-Díaz de Cerio}

Clínica Universidad de Navarra

\section{Laura Hato}

Clínica Universidad de Navarra

\section{Francisco Guillén-Grima}

Clínica Univseridad de Navarra

Oscar Fernández-Hidalgo

Clínica Universidad de Navarra

\section{Susana De La Cruz}

Complejo Hospitalario de Navarra

\section{María Dolores Lozano}

Clínica Universidad de Navarra

\section{Miguel A Idoate}

Clínica Universidad de Navarra

\section{Marta Santisteban}

Clínica Universidad de Navarra 
Keywords: Breast cancer, dendritic cell vaccines, TILs, neoadjuvant scenario, CD8 and triple negative. Posted Date: November 1st, 2021

DOI: https://doi.org/10.21203/rs.3.rs-643452/v3

License: (c) (1) This work is licensed under a Creative Commons Attribution 4.0 International License. Read Full License 


\section{Abstract}

Purpose: The addition of dendritic cell vaccines (DCV) to neoadjuvant chemotherapy (NAC) could induce immune biomarker changes in those patients with residual disease (RD) by transforming tumor microenvironment.

Methods: Core diagnostic biopsies and surgical specimens from 80 patients (38 in the Vaccinated Group plus NAC (VG) and 42 in the Control Group (CG) treated only with NAC) were selected. We quantify TILs (CD8, CD4 and CD45RO) using Immunohistochemistry (IHC) and the Automated Cellular Imaging System (ACIS III) in the core-diagnostic biopsies and in the surgical specimens, to compare the amount of TILs in each group.

Results: A CD8 rise in TNBC samples was observed after NAC plus DCV, changing from $4.48 \%$ in the biopsy to $6.70 \%$ in the surgical specimen, not reaching statistically significant differences $(p=0.11)$. TNBC patients in the CG showed a TILs drop from $2.71 \%$ in the biopsy to $0.18 \%$ in the surgical specimen $(p=0.5)$. We also found that $66.7 \%(4 / 6)$ of TNBC patients from VG registered an increase in TILs after treatment as compared to $20 \%(1 / 5)$ of TNBC patients in the CG $(p=0.24)$. This phenomenon is not observed in the other biologic subtypes. An association between before NAC CD8 TILs (4\% cut-off point) and pathological complete response in the VG was found in univariate and multivariate analysis (OR=1.41, IC95\% 1.05-1.90; $p=0.02$, and $O R=2.0$, IC95\% 1.05-3.9; $p=0.03$, respectively).

Conclusion: Our findings suggest that patients with TNBC especially benefit from the stimulation of the antitumor immune system by using DCV pulsed with tumor antigens.

\section{Background}

Immune infiltration in breast cancer (BC) milieu has driven to the achievement of a better outcome of these patients with standard chemotherapy, but also has enhanced the incorporation of immunotherapy as a therapeutic strategy. Thus, higher levels of tumor infiltrating lymphocytes (TILs) on the core diagnostic biopsy have been related to increased pathological complete responses (pCR) with neoadjuvant chemotherapy (NAC) and longer event-free survival (EFS) and overall survival (OS) in the more aggressive BC subtypes [1-5]. However, the presence of residual disease (RD) after NAC implies further adjuvant chemotherapy that could be avoided if $\mathrm{pCR}$ would have been reached. Additionally, the rise of TILs in RD after NAC has been linked to a better outcome and it could be considered a surrogate marker for long-term treatment efficacy in triple negative breast cancer (TNBC) patients in the absence of pCR [5-7].

Aside from these data, the development of new immune strategies that convert cold into hot immune enriched tumors are mandatory. The study of biological changes in BC patients with RD after NAC opens a translational window in breast tumors and its milieu in this population with a worse prognosis. Immune checkpoint inhibitors $(\mathrm{CPI})$ have been evaluated in $\mathrm{BC}$ with outstanding results in combination with chemotherapy in the neoadjuvant arena [8-14] although with an increased toxicity profile, and without 
information regarding biological impact on tumor milieu. Dendritic cells, as the main directors of the immune system, have been studied for their role in the antigenic cross presentation, a key step for T cytotoxic responses. Active cell therapy with dendritic vaccines (DCV) has shown tumor growth inhibition and $\mathrm{T}$ cell memory activation in preclinical models [15] as well as clinical improvement in BC patients without further toxicity in different scenarios $[16,17]$. So far, the implementation of DCV together with NAC in naïve $B C$ patients could be a big challenge by increasing host immunity and immunogenic cell death in tumoral cells and generating potential modifications in tumor milieu. Moreover, our center has already demonstrated prior experience with active DCV in other solid malignancies $[18,19]$

The aim of this study is to evaluate if the addition of DCV to NAC could induce immune biomarker changes in those patients with RD by transforming tumor microenvironment, in order to identify which group of patients could benefit from this strategy and to select the best therapy in the maintenance scenario in upcoming studies.

\section{Materials And Methods}

\section{Patients}

Patients in the vaccinated group (VG) were recruited from 2011 to 2015 in the phase II non-randomized multicentric clinical trial NCT01431196 and as compassionate use for DCV. All these patients were treated with the same NAC schedule consisting of 4 cycles of dose-dense epirubicin plus cyclophosphamide with G-CSF support sequenced to 4 cycles of docetaxel each 21 days according to standard protocols and with the addition of DCV during taxane therapy. The control group (CG) was obtained from an historic cohort (2008-2015) treated at our center in the same way but without vaccines. Demographic features were well balanced among both groups[20]. No adjuvant chemotherapy was prescribed. Surgical management was performed after NAC and was followed by radiation therapy \pm endocrine therapy if needed. In the adjuvant setting, patients in the VG also received intradermal DCV loaded with autologous tumor lysate as described in our previous work [17, 20].

\section{Samples and criteria for analysis}

Core-diagnostic biopsies and surgical specimens from 80 patients (38 in the VG and 42 in the CG were selected. Pathologists did not know if the samples belong to the VG or CG when they performed the quantification of TILs (blinded-study).

Among the CG, two patients had multifocal/multicentric tumors in the breast. We were able to quantify TILs according to the characteristics of the core-diagnostic biopsies in the CG as follows: CD8 (37 samples), CD4 (39 samples) and CD45RO (36 samples). With respect to the surgical specimen, we studied 35 specimens for each marker. Regarding VG; 35, 34 and 34 core-diagnostic biopsies were available for CD8, CD4 and CD45ro biomarker, respectively. Surgical specimens were 27, 28 and 26 for CD8, CD 4 and CD45R0 markers, respectively. Samples were classified by biological subtype according to the $14^{\text {th }}$ St Gallen International Breast Cancer Conference [21]. Total pCR was considered as no infiltrating 
residual tumor (ypT0/Tis ypN0) in both breast surgical specimen and lymph nodes according to American Joint Committee on Cancer $8^{\text {th }}$ Edition. Recommendations from the International Immunooncology Biomarker Working Group on BC were used in order to evaluate TILs in the stromal compartment related to tumoral cells. The denominator used to determine the percentage of stromal TILs was the area of stromal tissue occupied by lymphocytes, not the number of stromal cells [22]. Moreover, these recommendations were applied to immunohistochemistry to be able to measure CD8, CD 4 and CD45RO lymphocytes in the stromal tumor area. After NAC markers were only measured on the samples with RD. Pathologists have not quantified TILs in the surgical specimen from patients that reached pCR because it is not accurate to know if TILs were epitelial or stromal in this scenario. All the quantifications are in the stromal compoment, epitehlial quantifications are not shown. No data for tertiary lymphoid structures were included in the study.

\section{Immunohistochemistry}

Immunohistochemistry (IHC) was performed using formaldehyde-fixed and paraffin-embedded tissue sections from 3 to $4 \mathrm{~mm}$ thick. The tissue sections were stained by Autostainer Link48 using a monoclonal Mouse Anti-Human CD8 (Dako® Clone C8/144B), CD4 (Dako® Clone 4B12) and CD45RO (Dako® Clon UCHL1). Antigen retrieval was performed by PT link in high pH at 98 degrees for 5 minutes.

\section{Immunohistochemistry measurement and scoring}

IHC was quantified using Automated Cellular Imaging System (ACIS III) in both the diagnostic and the surgical samples $[23,24]$. TILs quantification according to the international working group in HE samples was obtained by three pathologist ( $\mathrm{LM}, \mathrm{AC}, \mathrm{MI})$ from two different services with variability. These results are not shown in the manuscript.

The ACIS III system is an automated, bright-field microscope with a patented image processing and analysis software based on color detection and pattern recognition to evaluate cells or tissue sections stained with IHC. Each slide was scanned at 10X. Stromal areas were evaluated according to tumor burden within each sample. A minimum area of $0.23 \mathrm{~mm}^{2}$ and a maximum area of $3.10 \mathrm{~mm}^{2}$ by sample with an average area of $1.50 \mathrm{~mm}^{2}$ were analyzed. The score of each sample was obtained dividing the number of brown pixels (stained by $\mathrm{IHC}$ ) by the total stromal area of the selected fields. Then, the result was transformed to a percentage value. Only areas with invasive breast carcinoma and without any processing defect were analyzed (Figure 1).

\section{Statistical analysis}

Non-continuous data were compared by the Chi-square test and Fisher test. Normality was tested using Shapiro-Wilks Test. The Mann - Whitney U-test and the Wilcoxon test were employed to study unpaired non-parametric variables and paired non-parametric variables, respectively. Sensitivity, specificity, predictive values, logistic regression and receiver operator characteristic (ROC) curves were calculated. Data were analyzed with the SPSS statistical software (version 20.0 for Windows),OpenEpi: Open Source 
Epidemiologic Statistics for Public Health, Version 3.01 and R software Version 4.0.1. P-values $\leq 0.05$ were considered significant, and $95 \%$ confidence intervals were calculated.

\section{Results}

\section{Patients}

Patients features, therapy as well as pCR are described in Table 1. Pathological CR was $\sim$ three times higher in the VG $(p=0.03)$. No differences were found by biologic subtypes (not shown). 
Table 1

Patient characteristics corresponding to control and vaccinated groups.

\begin{tabular}{|lll|}
\hline Characteristics & Control group & Vaccinated group (VG) \\
\hline & $(\mathbf{C G})$ & 38 \\
\hline Age ${ }^{\text {a }}$ & 42 & 45.68 \\
(range) & 55.31 & $(36.15-74.48)$ \\
\hline Menopause ${ }^{\text {b }}$ (\%) & $(26-84.35)$ & $28(71.8)$ \\
\hline Pre-menopause & $18(42.9)$ & \\
\hline Post-menopause & & $11(28.2)$ \\
\hline Lymph node status* $(\%)$ & $24(57.1)$ & $19(50)$ \\
\hline Negative & $24(53.3)$ & $19(50)$ \\
\hline Positive & & $10(26.3)$ \\
\hline Biological Subtype* $(\%)$ & $21(46.7)$ & $12(31.6)$ \\
\hline Luminal A & $14(31.1)$ & $16(42)$. \\
\hline Luminal B & & $10(26.3)$ \\
\hline Triple Negative & $18(40)$ & $28(73.7)$ \\
\hline Pathological CR*(\%) & $13(28.9)$ & \\
\hline Yes & $4(8.9)$ & \\
\hline No & $41(91.1)$ & \\
\hline
\end{tabular}

${ }^{a}$ Median values. ${ }^{b}$ According the number of patients. ${ }^{*}$ According the number of tumor samples. $\mathrm{pCR}$ pathological complete response. E: epirubicin; C: cyclophosphamide; D: docetaxel; CBDCA: carboplatin. 


\begin{tabular}{|lll|}
\hline Characteristics & Control group & Vaccinated group (VG) \\
$\mathbf{N}$ & $\mathbf{C G})$ & $\mathbf{3 8}$ \\
\hline Treatment Schedule (\%) & $\mathbf{4 2}$ & $35(92.10)$ \\
EC $\rightarrow$ D & $39(92.85)$ & $3(7.90)$ \\
CBDCA added to D & $3(7.15)$ & $382.46(352-409)$ \\
Total Dose (mgr) & $380.56(298-406)$ & $333.54(274-400)$ \\
E (mean, range) & $340.78(282-398)$ & $36(94.73)$ \\
D (mean,range) & $41(97.61)$ & $2(5.27)$ \\
Radiotherapy & $1(2.39)$ & \\
Yes & & \\
No & & \\
\hline $\begin{array}{l}\text { a Median values. } \\
\text { pathological complete response. E: epirubicin; C: cyclophosphamide; D: docetaxel; CBDCA: } \\
\text { carboplatin. }\end{array}$
\end{tabular}

\section{TILs quantification before and after chemotherapy and in paired samples}

Basal CD8 TILs before NAC were well balanced and expressed as percentage in the CG versus VG $(1.43 \%$ versus $1.33 \%$; $p=0.75)$; CD 4 (2.33\% versus $2.10 \% ; p=0.77)$ and CD45RO (1.11\% versus $0.78 \% ; p=0.74)$, respectively. These same results are also shown for RD (postNAC) in Table 2. As we explained earlier, all the patients in this study with a paired-sample analysis of TILs are those with RD in the surgical specimen (those who reached pCR are excluded). CD8 TILs decreased after NAC \pm DCV in tumors as compared to initial values as follows: median CD8 was $1.43 \%(0.03 \%-13.29 \%)$ before NAC as compared to median CD8 of $0.58 \%(0.03 \%-39.28 \%)$ after NAC $(p=0.51)$ in the CG; and $1.33 \%(0.19 \%-16.26 \%)$ versus $0.59 \%(0.14 \%-11.66 \%)(p=0.42)$ in the VG. The biologic subtype with the highest rate of CD8 TILs in paired samples in both groups was the TNBC $(p<0.0001)$. When data were analyzed according to biologic subtype, a trend to a CD8 rise in TNBC samples was observed after NAC plus DCV, changing from $4.48 \%$ (0.48-16.26) in the diagnostic biopsy to $6.70 \%(0.76-11.66)$ in the surgical specimen, not reaching statistically significant differences $(p=0.11)$. On the contrary, TNBC patients in the CG showed a TILS drop from $2.71 \%$ in the biopsy to $0.18 \%$ in the surgical specimen $(p=0.5)$. Differences in the CD 8 before and after NAC in both groups are described in Table 2. We also found that a $66.7 \%(4 / 6)$ of TNBC patients from VG registered an increase in TILs after treatment as compared with $20 \%(1 / 5)$ of TNBC patients in the CG $(p=0.24)$. This phenomenon is not observed in the other biologic subtypes and is clarified in Figure 2. 
Table 2

Quantification of CD8, CD4 and CD45RO TILs according to biologic subtypes in tumor samples.

\begin{tabular}{|c|c|c|c|}
\hline $\begin{array}{l}\text { TILs } \\
\text { CD8 in CG }\end{array}$ & PreNAC \% (range) & $\begin{array}{l}\text { PostNAC\% } \\
\text { (range) }\end{array}$ & p \\
\hline All subtypes & $1.43(0.03-13.29)$ & $0.58(0.03-39.28)$ & 0.51 \\
\hline LA & $0.89(0.29-3.66)$ & $0,59(0.19-2.23)$ & 0.79 \\
\hline LB & $1.98(0.03-8.49)$ & $0.81(0.10-17.09)$ & 0.69 \\
\hline TN & $2,71(0,03-13,29)$ & $0.18(0.03-39.28)$ & 0.50 \\
\hline \multicolumn{4}{|l|}{ CD8 in VG } \\
\hline All subtypes & $1.33(0.19-16.26)$ & $0.59(0.14-11.66)$ & 0.42 \\
\hline LA & $0.90(0.25-1.29)$ & $0.45(0.15-1.62)$ & 0.13 \\
\hline LB & $1.08(0.19-3.06)$ & $0.56(0.14-2.65)$ & 0.07 \\
\hline TN & $4.48(0.48-16.26)$ & $6.70(0.76-11.66)$ & 0.11 \\
\hline \multicolumn{4}{|l|}{ CD4 in CG } \\
\hline All subtypes & $2.33(., 04-21.56)$ & $0.47(0-38.29)$ & $0.04 *$ \\
\hline LA & $0.54(0.04-3.35)$ & $0.18(0-2.82)$ & 0.15 \\
\hline LB & $2.65(0.10-21.56)$ & $0.65(0.03-13.47)$ & 0.17 \\
\hline TN & $4.31(0.49-18.41)$ & $2,33(0.02-38.29)$ & 0.68 \\
\hline \multicolumn{4}{|l|}{ CD4 in VG } \\
\hline All subtypes & $2.10(0.01-58.91)$ & $0.61(0.04-19.92)$ & 0.24 \\
\hline LA & $0.62(0.01-2.61)$ & $0.25(0.04-1,89)$ & 0.20 \\
\hline LB & $1.09(0.15-9.88)$ & $0.46(0.05-11.21)$ & 0.33 \\
\hline TN & $10.44(1.18-58.91)$ & $7.71(0.45-19.92)$ & 0.91 \\
\hline \multicolumn{4}{|l|}{ CD45ro in CG } \\
\hline All subtypes & $1,11(0,03-15,86)$ & $0,31(0,01-24,44)$ & 0,24 \\
\hline LA & $0,34(0,03-1,79)$ & $0,15(0,01-2,79)$ & 0,37 \\
\hline LB & $1,94(0,05-15,86)$ & $0,72(0,03-24,44)$ & 0,30 \\
\hline
\end{tabular}

Results are expressed as median values with range. CG: Control Group; VG: vaccinated group; LA: luminal A, LB: luminal B, TN: Triple negative. *Statistical significance. 


\begin{tabular}{|llll|}
\hline $\begin{array}{l}\text { TILs } \\
\text { CD8 in CG }\end{array}$ & PreNAC \% (range) & $\begin{array}{l}\text { PostNAC\% } \\
\text { (range) }\end{array}$ & P \\
\hline TN & $1,29(0,04-8,74)$ & $0,52(0,27-2,78)$ & 1,00 \\
CD45ro in VG & & $0,29(0,02-25,58)$ & 0,34 \\
\hline All subtypes & $0,78(0,02-32,31)$ & $0,13(0,02-1,99)$ & 0,16 \\
\hline LA & $0,55(0,02-2,37)$ & $0,27(0,09-11,27)$ & 0,76 \\
\hline LB & $0,49(0,03-3,25)$ & $2,26(0,25-25,58)$ & 0,50 \\
\hline TN & $2,85(0,37-32,31)$ & & \\
\hline $\begin{array}{l}\text { Results are expressed as median values with range. CG: Control Group; VG: vaccinated group; } \text { LA: } \\
\text { luminal A, LB: luminal B, TN: Triple negative.*Statistical significance. }\end{array}$ & \\
\hline
\end{tabular}

Regarding CD4 TILs, a significant decreased infiltration was shown in the CG with the effect of NAC $(p=0.04)$ when compared paired-samples that was not present in the experimental group $(p=0.24)$ (Table 2).

\section{TILs in the core-diagnostic biopsy: identifying target population for DCV}

An association between CD8 TILs before NAC and PCR in VG (see Table 1 for pCR) was found in univariate and multivariate analysis $(O R=1.41$, IC95\% 1.05-1.90; $p=0.02$, and $O R=2.0, I C 95 \%$ 1.05-3.9; $\mathrm{p}=0.03$, respectively). However, this association disappeared in the $\mathrm{CG}$ (univariate analysis $\mathrm{OR}=1.18$, (IC95\% 0.90-1.55; $p=0.22)$; and in the multivariate analysis, OR=1.11 (IC95\% 0.68-1.81; $p=0.65)$ ). No association between CD4 or CD45RO before NAC and PCR in both univariate and multivariate analysis was observed in the CG (CD4 univariate analysis $O R=1.11$ (IC95\% 0.94-1.32; $\mathrm{p}=0.18$ ); CD4 multivariate analysis OR=1.09 (IC95\% 0.83-1.44; $p=0.51)$; in the VG CD4 univariate analysis OR=1.10 (IC95\% 0.98-1.23; $p=0.08)$; multivariate analysis $O R=0.96(I C 95 \% 0.79-1.17 ; p=0.71)$. Regarding the univariate analysis of CD45RO marker in the CG we found an OR=1.04 (IC95\% 0.78-1.40; $p=0.75$ ); in the multivariate analysis, an $\mathrm{OR}=0.87$ (IC95\% 0.51-1.49; $\mathrm{p}=0.62$ ) was shown; and in the VG univariate analysis an OR=1.05 (IC95\% $0.95-1.15 ; p=0.33$ ) was described; lastly, in the multivariate analysis in the experimental group the OR=0.82 (IC95\% 0.66-1.02; $p=0.07)$. The association between TILs and $p C R$ disappears when the samples are organized by biologic subtype.

Related to PCR, ROC curve establishes a cut-off point of $4 \%$ in diagnostic

samples for CD8 TILs score (Figure 3). With this cut-off point, a 92.6\% sensitivity (IC95\% 76.63-97.94), 75\% specificity (IC95\% 40.93-92.85), 92.6\% positive predicted value (PPV) (IC95\% 76.63-97.94) and a 75\% negative predicted value (NPV) (IC95\% 40.93-92.85) were obtained in the VG. Using the same cut-off point in the CG, a similar sensitivity of 87.9\% (IC95\%:72.67-95.18) and a PPV of 93.55\% (IC95\%: 79.28- 
98.21) was reached, although the specificity and NPV were 50\% (IC95\%: 15-85) and 33.33\% (IC95\%: 9.6$70)$, respectively. Differences between both groups were not statistically significant $(p=0.20)$.

Regarding outcome, we have calculated the percentage of patients that relapse regarding percentage of TILs at diagnosis with a median follow-up of 8-years. With the established $4 \%$ TILs cut-off in the diagnostic specimen, $12.5 \%$ (1/8) patients with $\geq 4 \%$ CD8 TILs in the VG and 16.6\% (1/6) in the CG have disease progression; among those patients with <4\% TILs, 7.4\% (2/27) patients in the VG and 19\% (8/42) in the $C G$ relapsed. There is a trend in the VG to decrease relapse but due to the small sample size there is not enough statistical power to get significance (Fisher test, $p=0.30$ ).

No comparable results were found with CD4 and CD45RO TILs.

\section{Residual Disease}

We also correlate quantification of TILs in residual samples with the level of pathological response categorized by Miller \& Payne ( 3 and 4 M\&P grades). Although sample size was pretty limited, we have not seen correlation among both variables (Spearman's rank correlation $\mathrm{P}=0.77$ ), although 23 vaccinated patients with residual disease reached moderate to high pathological responses ( 3 and 4 M\&P grades) compared to 4 patients with poorer pathological responses (1 and 2 M\&P grades).

We have not established a cut-off point in the RD related to outcome as we have performed within diagnostic biopsy, because our simple size is pretty limited. In fact, we have only 4 and 3 patients in the VG and CG respectively with $\geq 4 \%$ TILs. Results should not be reliable under these conditions.

\section{Discussion}

To our knowledge, this is the first report that identifies good responders to DCV in combination with NAC related to CD8 expression in the needle core biopsy with the established $4 \%$ cut-off point. Moreover, this is the first time that biomarker changes in the tumor and its milieu are reported in RD in naïve BC patients treated with standard NAC \pm active cell based-immunotherapy, by showing maintained CD4 levels and a trend to a rise in CD8 infiltration (only in TNBC). We have also appreciate a trend to a better outcome when TILs were higher in RD, with less relapse events in the VG. Clinical impact of these findings could be relevant to improve selection of: 1) patients that could benefit from the addition of DCV to NAC; and 2) the best maintenance therapy in TNBC subtypes with RD based on the expression of CD8 and CD4 markers to choose standard capecitabine $[8,25]$ versus immunotherapy. Modifications on the tumor niche that reflects immune activation after DCV helps to be open wide to less toxic and more specific immunotherapy.

Regarding TILs, TNBC patients reach higher levels than the luminal subtypes in our study, as described before [5]. This could be in part due to specific mutational signatures, copy number variations, stromal metagenes and clonal heterogeneity of TNBC subtype [26-28]. 
We have shown a non-significant increase in stromal TILs after combined therapies with NAC plus DCV in up to $66 \%$ of TNBC patients, but not in the luminal subtypes. Probably our results are not significant due to the small sample size, being one limitation, and conferring the study an exploratory role that should be validated in other cohorts of patients.

Increased TILS could illustrate a reinforcement of the immune niche produced by DCV in BC patients. It can be suggested that vaccines could produce an increase in CD8 T cells post-treatment in TNBC. Waks et al. found that luminal BC are less enriched in CD8 TILs than other BC subtypes and this cell population decreased after NAC [29]. Nowadays, there is not enough knowledge about how immunotherapy could change $\mathrm{BC}$ microenvironment. Results of the studies that have worked with paired samples (before and after conventional NAC and no immunotherapy) remain controversial, suggesting different roles of immune cell populations in carcinogenesis, response to therapies, tumor progression and a crosstalk among the tumor and the microenvironment. While some reports described a reduction of CD8 TILs in cancer milieu after NAC [29-32], others shown an increase on TILs count [6, 33, 34] or an inversion on the CD4/CD8 ratio [4]. Luen et al. described an increase in TILs level in $48 \%$ of the patients and a decreased in $47 \%$ of the patients in RD after NAC [35].

The input of immunotherapy in $\mathrm{BC}$ patients in the neoadjuvant scenario looks for a global improvement within the tumor (higher pCR), the niche (hottest tumors) and the systemic immune surveillance. Dendritic cell-based adjuvant immunotherapy has already shown a gain in CD8 T cells in peripheral blood in nonluminal BC with an encouraging PFS improvement, suggesting benefits in the systemic immunity [16]. Vaccination is more effective in the prevention of tumor growth, and probably the clinical advantage could be more relevant in patients with a small tumor burden and with a preserved immune system (naïve of therapies) than in large tumor burden patients, including metastatic scenario, and with an exhausted immune system. Thus, immune cell profile of primary versus metastases of $B C$ patients is different [36, 37].

Current efforts have been made to standardize the quantification of TILs and to produce reliable results [38-41]. Some authors pointed out that the reproducibility of TILs evaluation improves when the categories are simplified to low versus high TILs, based on the concept of lymphocyte-predominant breast cancer phenotype [42] but the standard cut-off points need to be defined. The incorporation of digital analysis (ACIS III) provided a better quantification of the stromal TILs avoiding interobserver bias. Additionally, other aspect to be considered is the selection of the area to be evaluated and how many fields should be selected. Recent works support the fact that the average lymphocyte score from a single biopsy of a tumor is reasonably representative of the whole cancer [43]. Our results showed that a cut-off point of $4 \%$ CD8 in the diagnostic specimen could predict a better pathologic response when DCV are added to conventional NAC, with a lower relapse (not significant). As it can be observed in Figure 1, this cut-off could be considered a relatively high density of CD8 cells in the stromal tumor. This optical microscope immunohistochemical image could be used as a reference of what we consider as the minimal density of cells which correlates with response to vaccines. 
Qingzhu et al., found in their meta-analysis that memory T lymphocyte infiltration of a tumor site could serve as a indicator for OS and DFS prediction in patients with malignant tumours [44]. Also, Yajima et al., find a relationship between high CD45RO (i.e memory $T$ lymphocyte and marker of activated T cells) expression and a lower pathological stage in BC patients [45]. The importance of CD4 TILs is not clear in BC scenario. No association between CD4 and pCR was found in our study, although in the CG CD4 TILs significantly decreased in patients after NAC $(p=0.04)$ as compared to those who received the experimental therapy which remained stable $(\mathrm{p}=0.24)$ (Table 2$)$. However, most of the studies correlate CD4 or CD4/CD8 ratio with an increased $\mathrm{pCR}$ and better survival [34, 5], and this fact strengths the role of DCV.

We have not look for tertiary lymphoid structures in this study although they are seen occasionally in the samples. Its neogenesis is not specific for tumors and immune therapies applied in oncology, so they also appear in autoimmune and infectious diseases, trasplanted solid organs, inflammatory disorders as well as after preventive vaccines (eg; HPV).

Although more studies are needed to establish the benefit of DCV addition to chemotherapy, our results suggest that we are moving in the right direction. Identification of predictive and prognostic biomarkers to select patients that could benefit from the addition of immunotherapy to the standard systemic chemotherapy is key to develop more efficient therapeutic strategies [47]. The relationship between lymphocyte-predominant breast cancer patients in the case of the TNBC [48] and the PCR, PFS and OS is clearly established [49-51]. Nonetheless, an outstanding selection of good responders to immunotherapy is complex [52] and the study of biomarkers in RD after NAC plus immunotherapy as well as in peripheral blood is mandatory to improve this limitation.

Evaluation of tumor responses to immune strategies by imaging techniques has become tricky because changes in tumor burden need new response criteria based on special guidelines (iRECIST) [53]. In this way, biologic markers in the blood, the tumor and its milieu could contribute to a more specific information than imaging markers regarding patient selection for immune strategies in the early BC arena.

In conclusion, a $4 \%$ cut-off point of CD8 TILs in a TNBC subtype could help to establish which patients can benefit from DCV added to NAC. In the same way, a maintained expression of CD4 as well as an increased infiltration of stromal CD8 after DCV therapy could improve responses to further therapies, as seen in other solid tumors after relapse to IMT strategy when patients were treated with second line chemotherapy in the advanced scenario [6, 7]. A trend to a lower relapse in diagnostic biopsies enriched with TILs has been also shown. Our findings suggest that patients with TNBC especially benefit from the stimulation of the antitumor immune system by using DCV pulsed with tumor antigens. Deeper studies based on immune profiling genomic panels in the tumor and immune cell populations on peripheral blood are still ongoing within our patients and could help us to elucidate the biological behavior of $\mathrm{BC}$ as well as the benefits from adding immunotherapy to conventional NAC. Promising combined immune 
approaches potentiating immune system with DCV added to the blockade of immune checkpoints together with NAC should be tested in clinical trials in this selected population.

\section{Declarations}

\section{Funding}

This work is supported by the Ministerio de Sanidad y Política Social in the section of Advanced Therapies [TRA-005] and by the Ministerio de Ciencia e Innovación [PI16/01245], Gobierno de España.

\section{Competing interest}

M. Santisteban has received honoraria from Roche, Pfizer and Novartis and travel

support from Roche, Pfizer and Miltenyi. A. López-Díaz de Cerio and S. Inogés have travel support from Miltenyi. No other disclosures were reported. All remaining authors have declared no conflict of interest.

\section{Availability of data and materials}

The datasets generated and analysed during the current study are available from the corresponding autor on reasonable request.

Code availability Not applicable.

\section{Authors' contributions}

L.M collected data, built the dataset, performed the analysis and helped write and revise the manuscript. A. L.J collected data and helped revise the manuscript. A. C collected data and helped revise the manuscript. P. S collected data and helped revise the manuscript. B.P.S helped to perform the statistical analysis and revise the manuscript. S.I made the dendritic cell vaccines and helped revise the manuscript. A.L.D.C. made the dendritic cell vaccines and helped revise the manuscript. F.G helped to perform the statistical analysis. O. F.H helped recruit patients and collect data. S.D.L.C helped recruit patients. L.H. collect data. MD. L collect data. M.I designed the samples assessment, collect data and helped write and revise the manuscript. M.S. designed the clinical trial, collected data and helped write and revise the manuscript.

\section{Ethics approval and consent to participate}

This study was approved by the Navarra University Ethics Committee

Consent for publication Not applicable.

\section{Acknowledgements}

We acknowledge Jaione García for her support and technical expertise in Immunohistochemistry. 


\section{References}

1. Savas $P$, Salgado R, Denkert C, et al (2016) Clinical relevance of host immunity in breast cancer: from TILs to the clinic. Nat Rev Clin Oncol 13:228-241. https://doi.org/10.1038/nrclinonc.2015.215

2. Ali HR, Provenzano E, Dawson S-J, et al (2014) Association between CD8+ T-cell infiltration and breast cancer survival in 12439 patients. Annals of Oncology 25:1536-1543. https://doi.org/10.1093/annonc/mdu191

3. Wang K, Xu J, Zhang T, Xue D (2016) Tumor-infiltrating lymphocytes in breast cancer predict the response to chemotherapy and survival outcome: A meta-analysis. Oncotarget 7:44288-44298. https://doi.org/10.18632/oncotarget.9988

4. García-Martínez E, Gil GL, Benito AC, et al (2014) Tumor-infiltrating immune cell profiles and their change after neoadjuvant chemotherapy predict response and prognosis of breast cancer. Breast Cancer Res 16:488. https://doi.org/10.1186/s13058-014-0488-5

5. Denkert C, von Minckwitz G, Darb-Esfahani S, et al (2018) Tumour-infiltrating lymphocytes and prognosis in different subtypes of breast cancer: a pooled analysis of 3771 patients treated with neoadjuvant therapy. The Lancet Oncology 19:40-50. https://doi.org/10.1016/S14702045(17)30904-X

6. Dieci MV, Criscitiello C, Goubar A, et al (2014) Prognostic value of tumor-infiltrating lymphocytes on residual disease after primary chemotherapy for triple-negative breast cancer: a retrospective multicenter study. Annals of Oncology 25:611-618. https://doi.org/10.1093/annonc/mdt556

7. Hwang HW, Jung $\mathrm{H}$, Hyeon J, et al (2019) A nomogram to predict pathologic complete response (pCR) and the value of tumor-infiltrating lymphocytes (TILs) for prediction of response to neoadjuvant chemotherapy (NAC) in breast cancer patients. Breast Cancer Res Treat 173:255-266. https://doi.org/10.1007/s10549-018-4981-x

8. Masuda N, Lee S-J, Ohtani S, et al (2017) Adjuvant Capecitabine for Breast Cancer after Preoperative Chemotherapy. N Engl J Med 376:2147-2159. https://doi.org/10.1056/NEJMoa1612645

9. Schmid P, Cortes J, Pusztai L, et al (2020) Pembrolizumab for Early Triple-Negative Breast Cancer. N Engl J Med 382:810-821. https://doi.org/10.1056/NEJMoa1910549

10. Nanda R, Liu MC, Yau C, et al (2020) Effect of Pembrolizumab Plus Neoadjuvant Chemotherapy on Pathologic Complete Response in Women With Early-Stage Breast Cancer: An Analysis of the Ongoing Phase 2 Adaptively Randomized I-SPY2 Trial. JAMA Oncol 6:676. https://doi.org/10.1001/jamaoncol.2019.6650

11. Mittendorf EA, Zhang $\mathrm{H}$, Barrios $\mathrm{CH}$, et al (2020) Neoadjuvant atezolizumab in combination with sequential nab-paclitaxel and anthracycline-based chemotherapy versus placebo and chemotherapy in patients with early-stage triple-negative breast cancer (IMpassion031): a randomised, double-blind, phase 3 trial. The Lancet 396:1090-1100. https://doi.org/10.1016/S0140-6736(20)31953-X

12. Loibl S, Untch M, Burchardi N, et al (2019) A randomised phase II study investigating durvalumab in addition to an anthracycline taxane-based neoadjuvant therapy in early triple-negative breast cancer: 
clinical results and biomarker analysis of GeparNuevo study. Annals of Oncology 30:1279-1288. https://doi.org/10.1093/annonc/mdz158

13. Martin-Romano P, Ammari S, El-Dakdoukti Y, et al (2020) Chemotherapy beyond immune checkpoint inhibitors in patients with metastatic colorectal cancer. European Journal of Cancer 137:117-126. https://doi.org/10.1016/j.ejca.2020.06.030

14. Dwary AD, Master S, Patel A, et al (2017) Excellent response to chemotherapy post immunotherapy. Oncotarget 8:91795-91802. https://doi.org/10.18632/oncotarget.20030

15. Fields RC, Shimizu K, Mulé JJ (1998) Murine dendritic cells pulsed with whole tumor lysates mediate potent antitumor immune responses in vitro and in vivo. PNAS 95:9482-9487. https://doi.org/10.1073/pnas.95.16.9482

16. Qi C-J, Ning Y-L, Han Y-S, et al (2012) Autologous dendritic cell vaccine for estrogen receptor (ER)/progestin receptor (PR) double-negative breast cancer. Cancer Immunology, Immunotherapy 61:1415-1424. https://doi.org/10.1007/s00262-011-1192-2

17. Trial With Autologous Dendritic Cell Vaccination in Patients With Stage II-III HER2 Negative Breast Cancer - Full Text View - ClinicalTrials.gov

18. Rodriguez J, Castañón E, Perez-Gracia JL, et al (2018) A randomized phase II clinical trial of dendritic cell vaccination following complete resection of colon cancer liver metastasis. j immunotherapy cancer 6:96. https://doi.org/10.1186/s40425-018-0405-z

19. Inogés S, Tejada S, de Cerio AL-D, et al (2017) A phase II trial of autologous dendritic cell vaccination and radiochemotherapy following fluorescence-guided surgery in newly diagnosed glioblastoma patients. J Transl Med 15:104. https://doi.org/10.1186/s12967-017-1202-z

20. Solans BP, López-Díaz de Cerio A, Elizalde A, et al (2019) Assessing the impact of the addition of dendritic cell vaccination to neoadjuvant chemotherapy in breast cancer patients: A model-based characterization approach. Br J Clin Pharmacol 85:1670-1683. https://doi.org/10.1111/bcp.13947

21. Coates AS, Winer EP, Goldhirsch A, et al (2015) Tailoring therapies-improving the management of early breast cancer: St Gallen International Expert Consensus on the Primary Therapy of Early Breast Cancer 2015. Annals of Oncology 26:1533-1546. https://doi.org/10.1093/annonc/mdv221

22. Salgado R, Denkert C, Demaria S, et al (2015) The evaluation of tumor-infiltrating lymphocytes (TILS) in breast cancer: Recommendations by an International TILS Working Group 2014. Annals of Oncology

23. Rojo MG, Bueno G, Slodkowska J (2010) Review of imaging solutions for integrated quantitative immunohistochemistry in the Pathology daily practice. Folia Histochem Cytobiol 47:349-354. https://doi.org/10.2478/v10042-008-0114-4

24. Słodkowska J, Filas V, Buszkiewicz E, et al (2010) Study on breast carcinoma Her2/neu and hormonal receptors status assessed by automated images analysis systems: ACIS III (Dako) and ScanScope (Aperio). Folia Histochem Cytobiol 48:19-25. https://doi.org/10.2478/v10042-010-00151 
25. Lluch A, Barrios CH, Torrecillas L, et al (2020) Phase III Trial of Adjuvant Capecitabine After Standard Neo-/Adjuvant Chemotherapy in Patients With Early Triple-Negative Breast Cancer (GEICAM/200311_CIBOMA/2004-01). JCO 38:203-213. https://doi.org/10.1200/JC0.19.00904

26. METABRIC Group, Curtis C, Shah SP, et al (2012) The genomic and transcriptomic architecture of 2,000 breast tumours reveals novel subgroups. Nature 486:346-352. https://doi.org/10.1038/nature10983

27. Bianchini G, Qi Y, Alvarez RH, et al (2010) Molecular Anatomy of Breast Cancer Stroma and Its Prognostic Value in Estrogen Receptor-Positive and -Negative Cancers. JCO 28:4316-4323. https://doi.org/10.1200/JC0.2009.27.2419

28. Wali VB, Langdon CG, Held MA, et al (2017) Systematic Drug Screening Identifies Tractable Targeted Combination Therapies in Triple-Negative Breast Cancer. Cancer Res 77:566-578. https://doi.org/10.1158/0008-5472.CAN-16-1901

29. Waks AG, Stover DG, Guerriero JL, et al (2019) The Immune Microenvironment in Hormone Receptor-Positive Breast Cancer Before and After Preoperative Chemotherapy. Clin Cancer Res 25:4644-4655. https://doi.org/10.1158/1078-0432.CCR-19-0173

30. Loi S, Dushyanthen S, Beavis PA, et al (2016) RAS/MAPK Activation Is Associated with Reduced Tumor-Infiltrating Lymphocytes in Triple-Negative Breast Cancer: Therapeutic Cooperation Between MEK and PD-1/PD-L1 Immune Checkpoint Inhibitors. Clin Cancer Res 22:1499-1509. https://doi.org/10.1158/1078-0432.CCR-15-1125

31. Pelekanou V, Barlow WE, Nahleh ZA, et al (2018) Tumor-Infiltrating Lymphocytes and PD-L1 Expression in Pre-and Posttreatment Breast Cancers in the SWOG S0800 Phase II Neoadjuvant Chemotherapy Trial. Mol Cancer Ther 17:1324-1331. https://doi.org/10.1158/1535-7163.MCT-171005

32. (2018) Mesenchymal-epithelial Transition and Tumor Vascular Remodeling in Eribulin Chemotherapy for Breast Cancer. AR 38:. https://doi.org/10.21873/anticanres.12236

33. Demaria S, Volm MD, Shapiro RL, et al (2001) Development of Tumor-infiltrating Lymphocytes in Breast Cancer after Neoadjuvant Paclitaxel Chemotherapy. Clin Cancer Res 7:3025-3030

34. Miyashita M, Sasano H, Tamaki K, et al (2015) Prognostic significance of tumor-infiltrating CD8+ and FOXP3+ lymphocytes in residual tumors and alterations in these parameters after neoadjuvant chemotherapy in triple-negative breast cancer: a retrospective multicenter study. Breast Cancer Res 17:124. https://doi.org/10.1186/s13058-015-0632-x

35. Luen SJ, Salgado R, Dieci MV, et al (2019) Prognostic implications of residual disease tumorinfiltrating lymphocytes and residual cancer burden in triple-negative breast cancer patients after neoadjuvant chemotherapy. Annals of Oncology 30:236-242. https://doi.org/10.1093/annonc/mdy547

36. Szekely B, Bossuyt V, Li X, et al (2018) Immunological differences between primary and metastatic breast cancer. Annals of Oncology 29:2232-2239. https://doi.org/10.1093/annonc/mdy399 
37. Finn OJ, Forni G (2002) Prophylactic cancer vaccines. Current Opinion in Immunology 14:172-177. https://doi.org/10.1016/S0952-7915(02)00317-5

38. Hendry S, Salgado R, Gevaert T, et al (2017) Assessing Tumor-Infiltrating Lymphocytes in Solid Tumors: A Practical Review for Pathologists and Proposal for a Standardized Method from the International Immuno-Oncology Biomarkers Working Group. Advances In Anatomic Pathology 24:311-335. https://doi.org/10.1097/PAP.0000000000000161

39. Jang N, Kwon HJ, Park MH, et al (2018) Prognostic Value of Tumor-Infiltrating Lymphocyte Density Assessed Using a Standardized Method Based on Molecular Subtypes and Adjuvant Chemotherapy in Invasive Breast Cancer. Ann Surg Oncol 25:937-946. https://doi.org/10.1245/s10434-017-6332-2

40. Swisher SK, Wu Y, Castaneda CA, et al (2016) Interobserver Agreement Between Pathologists Assessing Tumor-Infiltrating Lymphocytes (TILs) in Breast Cancer Using Methodology Proposed by the International TILs Working Group. Ann Surg Oncol 23:2242-2248. https://doi.org/10.1245/s10434-016-5173-8

41. Denkert C, Wienert S, Poterie A, et al (2016) Standardized evaluation of tumor-infiltrating lymphocytes in breast cancer: results of the ring studies of the international immuno-oncology biomarker working group. Mod Pathol 29:1155-1164. https://doi.org/10.1038/modpathol.2016.109

42. Tramm T, Di Caterino T, Jylling A-MB, et al (2018) Standardized assessment of tumor-infiltrating lymphocytes in breast cancer: an evaluation of inter-observer agreement between pathologists. Acta Oncologica. https://doi.org/10.1080/0284186X.2017.1403040

43. Mani NL, Schalper KA, Hatzis C, et al (2016) Quantitative assessment of the spatial heterogeneity of tumor-infiltrating lymphocytes in breast cancer. Breast Cancer Research 18:1-10. https://doi.org/10.1186/s13058-016-0737-x

44. Jia Q, Yang Y, Wan Y (2015) Tumor-infiltrating memory T-lymphocytes for prognostic prediction in cancer patients: a meta-analysis. Int J Clin Exp Med 8:1803-1813

45. Yajima R, Yajima T, Fujii T, et al (2016) Tumor-infiltrating CD45RO+ memory cells are associated with a favorable prognosis breast cancer. Breast Cancer 23:668-674. https://doi.org/10.1007/s12282015-0622-y

46. Gu-Trantien C, Loi S, Garaud S, et al (2013) CD4+ follicular helper T cell infiltration predicts breast cancer survival. J Clin Invest 123:2873-2892. https://doi.org/10.1172/JCl67428

47. Angell H, Galon J (2013) From the immune contexture to the Immunoscore: The role of prognostic and predictive immune markers in cancer. Current Opinion in Immunology

48. Hennigs A, Riedel F, Gondos A, et al (2016) Prognosis of breast cancer molecular subtypes in routine clinical care: A large prospective cohort study. BMC Cancer 1-9. https://doi.org/10.1186/s12885016-2766-3

49. Ibrahim EM, Al-Foheidi ME, Al-Mansour MM, Kazkaz GA (2014) The prognostic value of tumorinfiltrating lymphocytes in triple-negative breast cancer: a meta-analysis. Breast Cancer Res Treat 148:467-476. https://doi.org/10.1007/s10549-014-3185-2 
50. Fridman WH, Pagès F, Sautès-Fridman C, Galon J (2012) The immune contexture in human tumours: impact on clinical outcome. Nat Rev Cancer 12:298-306. https://doi.org/10.1038/nrc3245

51. Loi S, Sirtaine N, Piette F, et al (2013) Prognostic and Predictive Value of Tumor-Infiltrating Lymphocytes in a Phase III Randomized Adjuvant Breast Cancer Trial in Node-Positive Breast Cancer Comparing the Addition of Docetaxel to Doxorubicin With Doxorubicin-Based Chemotherapy: BIG 0298. JCO 31:860-867. https://doi.org/10.1200/JC0.2011.41.0902

52. Emens LA (2008) Cancer vaccines: on the threshold of success. Expert Opinion on Emerging Drugs 13:295-308. https://doi.org/10.1517/14728214.13.2.295

53. Seymour L, Bogaerts J, Perrone A, et al (2017) iRECIST: guidelines for response criteria for use in trials testing immunotherapeutics. The Lancet Oncology 18:e143-e152.

https://doi.org/10.1016/S1470-2045(17)30074-8

\section{Figures}

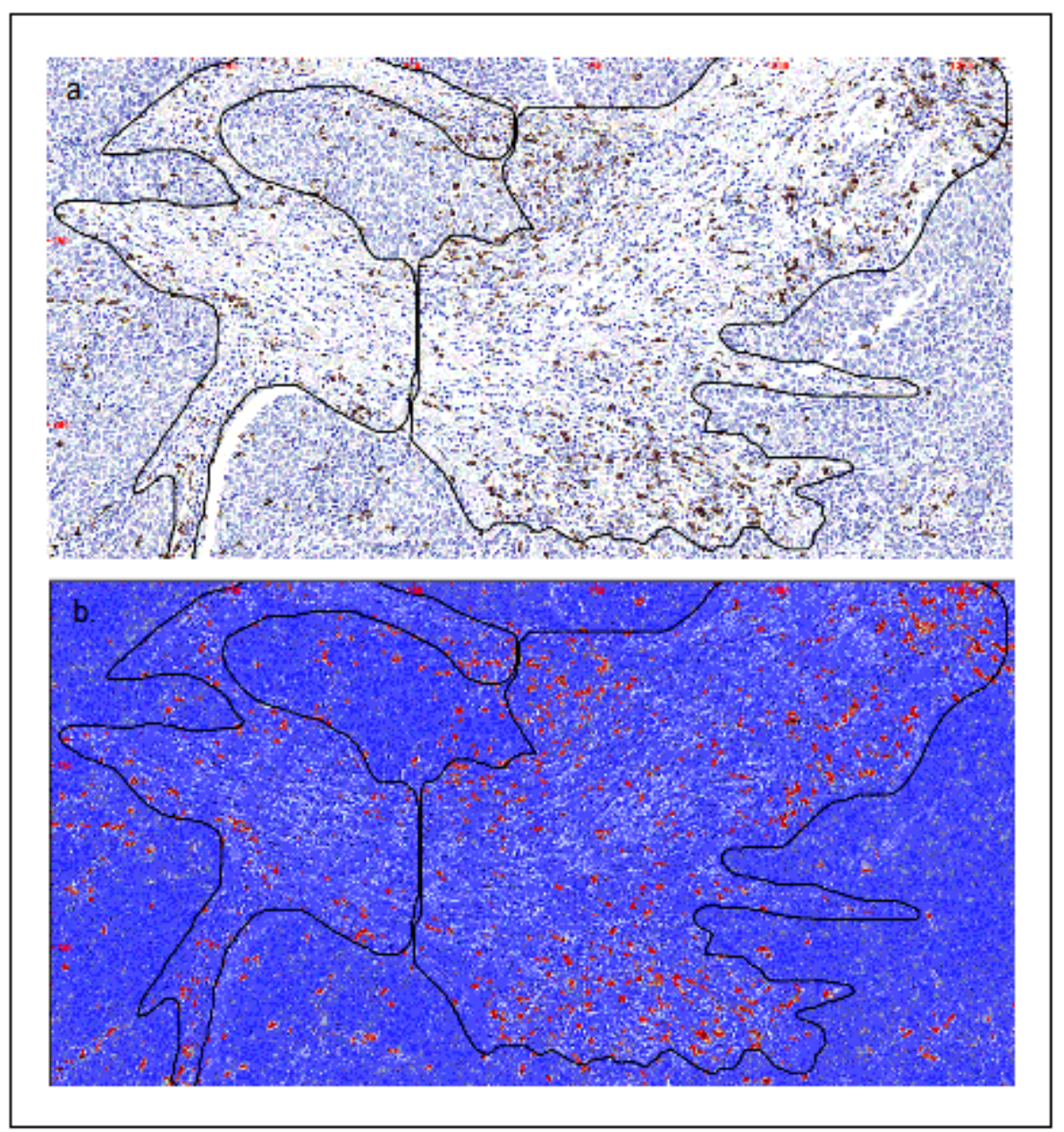

\section{Figure 1}

Immunohistochemistry measurement and scoring with ACIS III in a breast cancer sample. A) Image analysis by ACIS III of a biopsy, two stromal areas selected $(0.43 \mathrm{~mm} 2)$. B) TILs quantification by ACIS III, 
in red CD8 lymphocytes 5\%. Scanned at 10X. Magnification of 50\%.

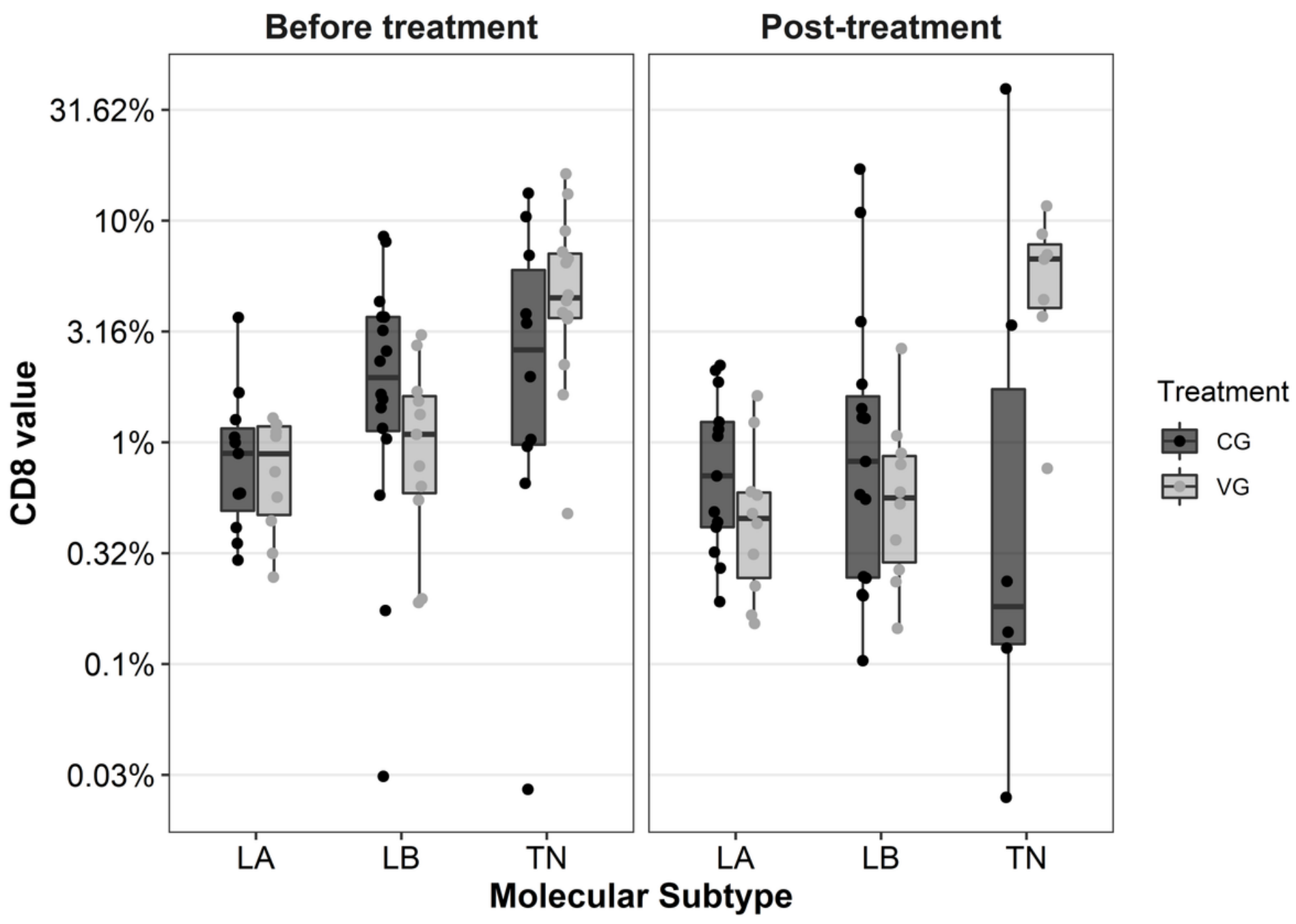

Figure 2

Boxplots diagram with the distribution of CD8 TILs before and after neoadjuvant chemotherapy according to biological subtype and group of treatment. Each dot represents the value for each patient. Yaxis is shown in logarithmic scale. TILs: tumor infiltrating lymphocytes, LA: luminal A, LB: luminal B, TN: triple negative BC, CG: control group, VG: vaccinated group. 

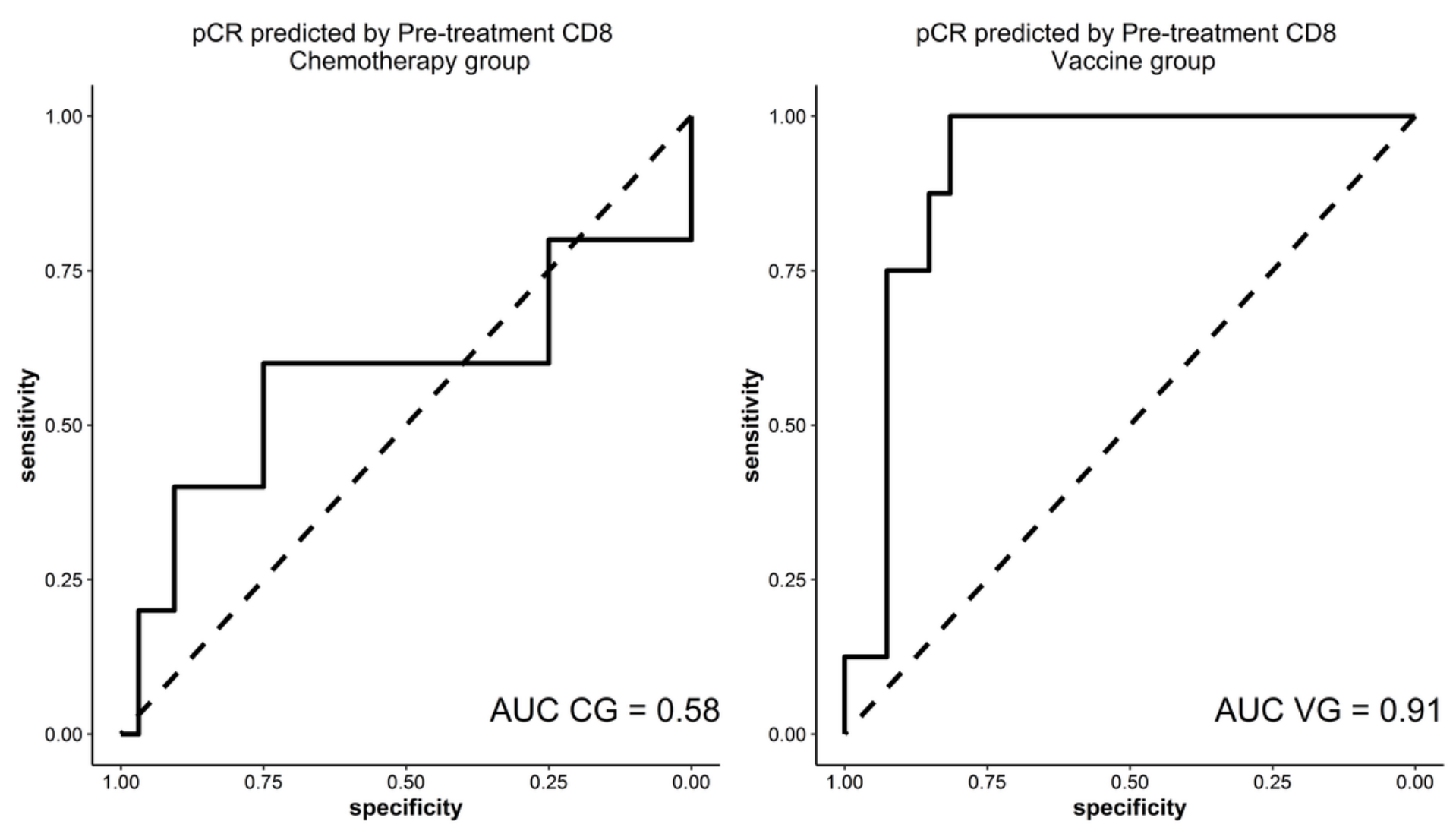

Figure 3

ROC curves in both therapeutic groups that correlate CD8 TILs in the diagnostic specimen and pCR. TILs: tumor infiltrating lymphocytes, pCR: pathological complete response, AUC: area under the curve, CG: control group, VG: vaccinated group. 